Review

\title{
Involving service users in health and social care research
}

Edited by Lesley Lowes and Ian Hulatt,

London: Routledge, 2005, pp. 214,

ISBN 0415346479

This book is an anthology by more than 40 contributors from consumer groups, families and parents, and professionals from social care, health care, and research areas. The contents are as mixed as the authors, from personal reflections, discussions on ethics, ideas for changes, to descriptions of actually fulfilled projects and their impact.

The first chapter sets the tone, "Theory and practice of user involvement in research: making the connection with public policy and practice." The last chapter ties it all together, "Working together to undertake research." The 15 chapters in between cover a wide range of research methods and projects, all of them involving users.

The basic idea of this book is that service users and professionals need to be partners in services. This partnership should also be reflected in research programmes. The research areas involved are medical sociology and health services research.

A couple of interesting issues emerged during reading. The first is post-research support. One respondent in a survey said, "I have taken part in research before, but nothing changed as a result of such research." Therefore, she was unwilling to participate in the current study. The author concludes that, "We need to ensure that findings of research are fed back and that findings are utilized ...". I find this experience in line with my own: it is often hard or even impossible to get people to take part in a new project if the previous had no visible impact, and was only a nuisance.

Another interesting experience is the difficulty with outcome-focused research: users evaluating social care-how can that be done? There is no "control situation", no "control group"; it all has to reflect what was actually experienced. There are few criteria for "good" or "bad" outcomes in social care or the caring in health care. In this book, several studies are focusing on the user perspective, which often has other angles than the professional. It reflects the conflict between process and outcome in quality research. Service users often take the outcome for granted and are more focused on the service issues, while professional groups are aiming at a certain outcome: the process is less important as long as the results are acceptable.

Several chapters describe the involvement of users from vulnerable groups, people with ill mental health, people with learning difficulties, children and older women. Some of the issues are the difficulty of being taken seriously as a service user, the negative attitude to service users as participants, and the politics behind the scene, "the hidden agenda". In the book, advice is given and methods proposed for partnership rather than the top-down methods.

For IJIC, the integration between service users and service providers in formal research projects, designed, processed, evaluated, and implemented into routine services is a new perspective. This book is an eye-opener, an inspiration, and a hand-book all at the same time. It will be most useful for policy-makers and researchers in health and social care involving marginalized, underprivileged and vulnerable user groups. The book may also be of interest as a textbook in university level studies within the care sciences.

Ingvar Karlberg, $\mathrm{MD}, \mathrm{PhD}$ Professor of Health Services Research, Göteborg University 